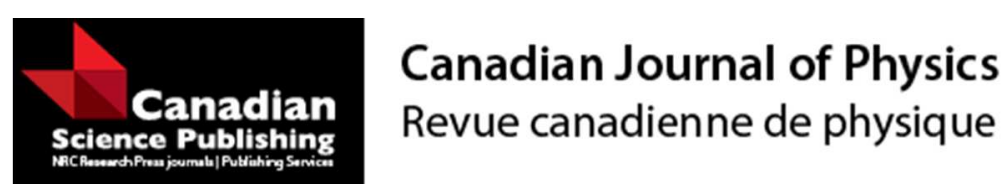

\title{
Simultaneous emergence of curved spacetime and quantum mechanics
}

\begin{tabular}{|r|l|}
\hline Journal: & Canadian Journal of Physics \\
\hline Manuscript ID & cjp-2015-0454.R1 \\
\hline Manuscript Type: & Article \\
\hline Date Submitted by the Author: & 04-Nov-2015 \\
\hline Complete List of Authors: & $\begin{array}{l}\text { De, S.S.; Calcutta University, Mathematics } \\
\text { Rahaman, F.; Jadavpur University, Mathematics }\end{array}$ \\
\hline Keyword: & $\begin{array}{l}\text { quantum mechanics, Emergent of curved spacetime, spinor, finsler } \\
\text { geomery, GENERALIZED UNCERTAINTY OPRINCIPLE }\end{array}$ \\
\hline
\end{tabular}

SCHOLARONE ${ }^{\text {IM }}$

Manuscripts 


\title{
Simultaneous emergence of curved spacetime and quantum mechanics
}

\author{
S S De ${ }^{1}$ and F Rahaman ${ }^{2}$ \\ ${ }^{1}$ Department of Applied Mathematics, University of Calcutta, Kolkata - 700009, India \\ E-mail: ssddadai08@rediffmail.com \\ ${ }^{2}$ Department of Mathematics, Jadavpur University, Kolkata - 700032, India \\ E-mail: rahaman@iucaa.ernet.in
}

November 13, 2015

\begin{abstract}
It is shown in this paper that the geometrically structure-less spacetime manifold is converted instantaneously to a curved one, the Riemannian or may be a Finslerian spacetime with an associated Riemannian spacetime, on the appearance of quantum Weyl spinors dependent only on time in that background flat manifold and having the symplectic property in the abstract space of spinors. The scenario depicts simultaneous emergence of the gravity in accord with general relativity and quantum mechanics. The emergent gravity leads to the generalized uncertainty principle, which in turn, ushers in discrete space time. The emerged space time is specified here as to be Finslerian and the field equation in that space time has been obtained from the classical one due to the arising quantized space and time. From this field equation we find the quantum field equation for highly massive (of the Planck order) spinors in the associated Riemannian space of the Finsler space, which is in fact, the background homogeneous and isotropic FRW space time of the universe. These highly massive spinors provide the mass distribution complying Einstein equivalence principle. All these occurred in the indivisible minimum time considered as zero time or spontaneity.
\end{abstract}

Pacs No.: 04.60.-m , 98.80.-k, 98.80.Jk

\section{Introduction}

One of the crucial problems in contemporary theoretical physics is to unify the four known interaction forces. After the advent of the Grand Unified Theories (GUT) the problem is now shifted in establishing a successful relation between the relativistic quantum field theories formulated on Minkowski space time, which are providing the conceptual framework of standard model of particle physics and the general covariance of space time in gravity according to the general relativity $(\mathrm{G} R$ ). In fact, to achieve a veritable unification of $\mathrm{G} R$ and Quantum mechanics ( $\mathrm{Q}$ ) it may not be necessary to have any presupposition of geometrical structure in the space time manifold or even smoothness of it. Also the physics of the Planck regime of the universe should be understood for this purpose. Thus 
the problem is that how the gravity might have been emerged as the metric structure of space time manifold in the usual setting of G R (being the origin of the Einstein equivalence principle).

For establishing the unification and also accomplishing quantization of gravity, there are several approaches such as string theory, loop quantum theory, theories based on discreteness of space time [1-9]. In these theories either fundamental status of $\mathrm{Q} \mathrm{M}$ or that of gravity has been postulated. Consequently, we have to encounter another important problem closely related to the unification of $G$ $\mathrm{R}$ and $\mathrm{Q}$ M. This is being to ascertain the fundamental status to any one of $\mathrm{G} \mathrm{R}$ and $\mathrm{Q} \mathrm{M}$ or to both of them.In case one of them is fundamental, the other must be emergent. But a perfect unification should demand that both of them must be fundamental as well as emergent. Consequently, we have to encounter another important problem closely related to the unification of $\mathrm{G} \mathrm{R}$ and $\mathrm{Q}$ M. This is being to ascertain the fundamental status to any one of $\mathrm{G} R$ and $\mathrm{Q}$ M,or to both of them.In case one of them is fundamental, the other must be emergent. But a perfect unification should demand that both of them must be fundamental as well as emergent. Also, we have to specify the actual phenomenon of their emergence, preferably, simultaneous emergence. To this end, it is presently proposed here that neither of Q M and gravity can be taken as to be fundamental, and on the contrary, their simultaneous and spontaneous emergence has been considered in a geometrically structure-less background space time manifold with the appearance of quantum Weyl spinors dependent only on time and having a symplectic property in the abstract space of spinors. In fact, apparition of these spinors can instantaneously convert the space time manifold into a metric structured one as well as to a discrete- structured space time manifold having minimum space and time lengths, all of the Planck scale. Thus, gravity in the setting of $\mathrm{G} \mathrm{R}$ emerges together with Q M manifested by the quanta of space and time and also by the quantum spinors. The consequence of the minimum time and space lengths is the generalized uncertainty principle (G U P) and vice versa. That is, the Heisenberg uncertainty principle is to be modified to $\mathrm{G} \mathrm{U} \mathrm{P}$ due to the emergent gravity and this must lead to the minimum time and space lengths. In this indivisible minimum time both $\mathrm{Q} M$ and $\mathrm{G} R$ emerged and after this quantum of time considered as "instantaneous", the time discreteness has no bearing on the subsequent evolution of the universe. Highly massive particles might have been generated in this Planck order minimum time and made it possible to comply Einstein equivalence principle.

We begin in section II, with the discussion on the emergence of structured space time due to the appearance of Weyl spinors in that flat space time manifold. In section III, identification of the emerged space time has been made. In section IV, in an alternative approach, the metric tensor of this space time is constructed from the basis spinors. In section $\mathrm{V}$, a brief discussion has been made on GUP as originated from gravitational interaction of photon with electron. We discuss in section VI, the appearance of highly massive particles as obtained from the field equation in associated Riemannian space, which is derived from the field equation in the emerged Finsler space. In final section VII the full scenario is presented with some concluding remarks.

\section{EMERGENCE OF STRUCTURED SPACE TIME :}

It is assumed here the appearance of two independent two-dimensional spinor fields depending only on time in the flat background space time manifold. The space of the two dimensional spinors is supposed to be endowed with a symplectic structure induced by a skew symmetric scalar product. This spinors $\psi^{(1)}$ and $\psi^{(2)}$ are in fact Weyl spinors, that is, they satisfy Weyl equation. If these spinors would have been dependent on both the space and time coordinates of the manifold, then the manifold may remain geometrically structure-less or flat. On the other hand, if they depend only on time, then the space time manifold must spontaneously have a curved structure, and consequently a non zero correction to the spinor fields arises. With this non zero correction $\Gamma_{\mu}$ or the generalized derivative (covariant derivative) $D_{\mu}$, we have the Weyl equation as the desired equation for the spinors in the curved space time. Here, the symplectic structure in the two dimensional spinor space, as induced by the skew-symmetric scalar product denoted by [.,..] is to be understood as the relation

$$
[\phi, \chi]=-[\chi, \phi]
$$


must remain valid for the spinors $\phi, \chi$. This scalar product can be expressible by a matrix

$$
\epsilon_{\alpha \beta}=\left(\begin{array}{cc}
0 & 1 \\
-1 & 0
\end{array}\right)
$$

and with the components of the spinors (referred to a certain basis). The spinors are expressed as

$$
\phi=\left(\begin{array}{l}
\phi^{1} \\
\phi^{2}
\end{array}\right), \chi=\left(\begin{array}{l}
\chi^{1} \\
\chi^{2}
\end{array}\right)
$$

The scalar product is

$$
[\phi, \chi]=\epsilon_{\alpha \beta} \phi^{\alpha} \chi^{\beta}=\left(\begin{array}{cc}
\phi^{1} & \chi^{1} \\
\phi^{2} & \chi^{2}
\end{array}\right) .
$$

The matrix (2) raises or lowers the indices of the spinors as follows:

$$
\phi^{\alpha}=\epsilon^{\alpha \beta} \phi_{\beta}, \phi_{\alpha}=\epsilon_{\alpha \beta} \phi^{\beta}
$$

where

$$
\epsilon^{\alpha \beta}=\left(\begin{array}{cc}
0 & -1 \\
1 & 0
\end{array}\right)=\epsilon_{\alpha \beta}^{T}=\epsilon_{\alpha \beta}^{-1} .
$$

Also, we have

$$
[\phi, \chi]=-\epsilon^{\alpha \beta} \phi_{\alpha} \chi_{\beta}=-[\chi, \phi]
$$

As it is said earlier, the two independent spinors $\psi^{(1)}, \psi^{(2)}$ are Weyl spinors and satisfy the Weyl equation. This Weyl equation can be obtained from the following Dirac equation in the curved space time for a massless particle or field [10-12 ] as

$$
i \gamma^{a} e_{a}^{\mu}\left(\partial_{\mu}+\hat{\Gamma_{\mu}}\right) \hat{\psi(i)}=0
$$

where $e_{a}^{\mu}$ denotes the vierbein and $\gamma^{\mu}$ are related to the flat space $\gamma^{a}$ matrices by the relation $\gamma^{\mu}=e_{a}^{\mu} \gamma^{a}$. The spin connection $\hat{\Gamma_{\mu}}$ is given by

$$
\hat{\Gamma_{\mu}}=\frac{1}{2} e_{h}^{\gamma} \nabla_{\mu} e_{\gamma k} S^{h k}=\frac{1}{2} e_{h}^{\gamma}\left(\partial_{\mu} e_{\gamma k}-\Gamma_{\mu \nu}^{\sigma} e_{\sigma k}\right) S^{h k}
$$

Here, $S^{h k}=\frac{1}{4}\left[\gamma^{h}, \gamma^{k}\right]$ is the skew symmetric generator of the Lorentz transformation for the spinor field and $\Gamma_{\mu \nu}^{\sigma}$ is the affine connection. By choosing the Dirac-Pauli representation of $\gamma^{a}$ matrices as

$$
\gamma^{0}=\left(\begin{array}{cc}
1 & 0 \\
0 & -1
\end{array}\right), \gamma^{i}=\left(\begin{array}{cc}
0 & -\sigma^{i} \\
\sigma^{i} & 0
\end{array}\right) ; i=1,2,3,
$$

where $\sigma^{i}$ are Pauli matrices, we can find after a straight forward calculation the following Weyl equations for the Weyl spinor field $\psi^{(i)}$ by writing the Dirac spinor $\hat{\psi^{(i)}}$ as $\hat{\psi^{(i)}}=\left(\begin{array}{c}\psi^{(i)} \\ -\psi^{(i)}\end{array}\right)$.

It is given as

$$
\sigma^{a} e_{a}^{\mu}\left(\partial_{\mu}+\Gamma_{\mu}\right) \psi^{(i)}=0
$$

where $\sigma^{0}=1$ and

$$
\Gamma_{\mu}=-\frac{1}{4}\left[\sum_{h, k=1,2,3} e_{h}^{\beta} \nabla_{\mu} e_{\beta k} \sigma^{h} \sigma^{k}+\sum_{k=1,2,3} \sigma^{k}\left(e_{k}^{\beta} \nabla_{\mu} e_{\beta 0}-e_{0}^{\beta} \nabla_{\mu} e_{\beta k}\right)\right] .
$$

The Weyl equation can also be written as

$$
i \sigma^{\mu}\left(\partial_{\mu}+\Gamma_{\mu}\right) \psi^{(i)}=0,
$$

where $\sigma^{\mu}=e_{a}^{\mu} \sigma^{a}$. 
By choosing the representation of $\gamma^{a}$ matrices as

$$
\gamma^{0}=\left(\begin{array}{cc}
1 & 0 \\
0 & -1
\end{array}\right), \quad \gamma^{i}=\left(\begin{array}{cc}
0 & \sigma^{i} \\
-\sigma^{i} & 0
\end{array}\right), i=1,2,3
$$

we can have the Weyl equation as

$$
i\left(\sigma^{0} e_{0}^{\mu}-\sum_{k=1,2,3} \sigma^{k} e_{k}^{\mu}\right)\left(\partial_{\mu}+\Gamma_{\mu}\right) \psi^{(i)}=0
$$

where

$$
\Gamma_{\mu}=-\frac{1}{4}\left[\sum_{h, k=1,2,3} e_{h}^{\beta} \nabla_{\mu} e_{\beta k} \sigma^{h} \sigma^{k}-\sum_{k=1,2,3} \sigma^{k}\left(e_{k}^{\beta} \nabla_{\mu} e_{\beta 0}-e_{0}^{\beta} \nabla_{\mu} e_{\beta k}\right)\right]
$$

For the case of $\Gamma_{a}=0, \psi^{(i)}$ must depend on both the space and time co ordinates of the manifold and vice versa, that is, space and time dependent spinors can satisfy Weyl equation with vanishing $\Gamma_{a}$ term. In this case, the flat space time manifold remains flat. On the contrary, if $\psi^{(i)}$ depends only on time (but not a constant), then it must have to satisfy Weyl equation with the non zero $\Gamma_{a}$, which can also be written ( follows from both the equations (12) and (14))

$$
i \sigma^{0} \partial_{0} \psi_{\alpha}^{(i)}+i \sigma^{a} \Gamma_{a \alpha \beta} \psi_{\beta}^{(i)}=0, i=1,2
$$

since $\psi_{\alpha}^{(i)}$ depends only on time $x^{0}=c t$. The non zero $\Gamma_{a}$ implies that the space time manifold must have changed to a curved one.

In this connection it is to be mentioned here that Kober (2008) has constructed "connection" of the spinor fields by defining a covariant derivative in such a way that it leaves two independent space time dependent basis spinors constant. With this covariant derivative the basis spinor fields automatically satisfy Weyl equation in curved space time. Also a tetrad formulation and metric with Minkowski signature have been constructed from the spinors having symplectic structure in the abstract space of spinors. But the resulted curved space time manifold may not have the same covariant derivative (as define therein ), that is, corresponding curved space time of the covariant derivative may not be the curved space time obtained from tetrad formulation. In fact, it was there only a presupposition of metric structure for the space time manifold in which the "connection" or covariant derivative are all defined.

By using symplectic condition (1) for the spinors $\psi^{(1)}$ and $\psi^{(2)}$, we can have from the equation (15) as

$$
\sigma^{a} \Gamma_{a \alpha \beta}=\frac{\partial_{0} \psi_{\alpha}^{(i)} \psi^{(2) \beta}-\partial_{0} \psi_{\alpha}^{(2)} \psi^{(1) \beta}}{\left[\psi^{(1)}, \psi^{(2)}\right]}
$$

where $\psi_{\alpha}^{(1)}, \psi^{(1) \beta}$ etc are related by $\epsilon_{\alpha \beta}$ as in (5). Here, $\sigma^{a} \Gamma_{a \alpha \beta}$ is dependent on the spinors $\psi^{(1)}$ and $\psi^{(2)}$ and has the similar form for the spin connection defined by [13].

\section{SPECIFICATION OF THE EMERGED SPACE TIME :}

The independent spinors $\psi^{(1)}$ and $\psi^{(2)}$ are specifically taken as $q\left(x^{0}\right)\left(\begin{array}{l}1 \\ 0\end{array}\right)$ and $q\left(x^{0}\right)\left(\begin{array}{l}0 \\ 1\end{array}\right)$ (referred to certain basis) respectively. These are dependent only on time $x^{0}$ and we have 


$$
\psi^{(1)}=\left(\begin{array}{c}
\psi_{1}^{(1)} \\
\psi_{2}^{(1)}
\end{array}\right)=\left(\begin{array}{c}
q\left(x^{0}\right) \\
0
\end{array}\right) \text { and } \psi^{(2)}=\left(\begin{array}{c}
\psi_{1}^{(2)} \\
\psi_{2}^{(2)}
\end{array}\right)=\left(\begin{array}{c}
0 \\
q\left(x^{0}\right)
\end{array}\right) .
$$

These Weyl spinors are also the spinors in the sense of Cartan [14]. A spinor $\left(\xi_{0}, \xi_{1}\right)$ is a spinor according to Cartan if

$$
x_{1}^{2}+x_{2}^{2}+x_{3}^{2}=0
$$

where

$$
x_{1}=\xi_{0}^{2}-\xi_{1}^{2}, x_{2}=i\left(\xi_{0}^{2}+\xi_{1}^{2}\right)
$$

and

$$
x_{3}=-2 \xi_{0} \xi_{1},
$$

for

$$
\psi^{(1)}=\xi_{0}=q\left(x^{0}\right) \text { and } \xi_{1}=0
$$

Consequently,

$$
x_{1}=q\left(x^{0}\right)^{2}, x_{2}=i q\left(x^{0}\right)^{2}, x_{3}=0 .
$$

Therefore,

$$
x_{1}^{2}+x_{2}^{2}+x_{3}^{2}=0 .
$$

Similarly, we see that $\psi^{(2)}$ is a spinor according to Cartan. In fact, any two component Weyl spinor is a Cartan spinor too.

Now, using (5), we have

$$
\left(\begin{array}{l}
\psi^{(1) 1} \\
\psi^{(1) 2}
\end{array}\right)=\left(\begin{array}{c}
0 \\
q\left(x^{0}\right)
\end{array}\right) \text { and }\left(\begin{array}{l}
\psi^{(2) 1} \\
\psi^{(2) 2}
\end{array}\right)=\left(\begin{array}{c}
-q\left(x^{0}\right) \\
0
\end{array}\right) .
$$

Then we have from (16)

$$
\sigma^{a} \Gamma_{a 11}=-\frac{q^{\prime}\left(x^{0}\right)}{q\left(x^{0}\right)}, \sigma^{a} \Gamma_{a 22}=-\frac{q^{\prime}\left(x^{0}\right)}{q\left(x^{0}\right)}, \sigma^{a} \Gamma_{a 12}=\sigma^{a} \Gamma_{a 21}=0 .
$$

Therefore,

$$
\left(\sigma^{a} \Gamma_{a \alpha \beta}\right)=-\frac{q^{\prime}\left(x^{0}\right)}{q\left(x^{0}\right)}\left(\begin{array}{ll}
1 & 0 \\
0 & 1
\end{array}\right)=-\frac{q^{\prime}\left(x^{0}\right)}{q\left(x^{0}\right)} I .
$$

Then the Weyl equation (15) becomes

$$
\sigma^{0} \partial_{0} \psi_{\alpha}^{(i)}-\frac{q^{\prime}\left(x^{0}\right)}{q\left(x^{0}\right)} \delta_{\alpha \beta} \psi_{\beta}^{(i)}=0
$$

or

$$
\sigma^{0} \partial_{0} \psi_{\alpha}^{(i)}-\frac{q^{\prime}\left(x^{0}\right)}{q\left(x^{0}\right)} \psi_{\alpha}^{(i)}=0, i=1,2
$$

This equation represents the Weyl equation in the emerged curved space time due to the appearance of two independent spinors depending only on time. But the exact nature of the metric structure of the manifold is yet to be determined.This can be accomplished by a comparison with the Weyl equation in a specific Riemannian space time manifold. Here we find the Weyl equation for the homogeneous and isotropic FRW spacetime ( with flat space ). The metric tensor for this spacetime is given by

$$
g_{00}=1, g_{i j}=-a^{2} \delta_{i j} .
$$


The vierbien and inverse vierbein fields are given by

$$
e_{0}^{\mu}=\delta_{0}^{\mu}, e_{i}^{\mu}=\frac{1}{a} \delta_{i}^{\mu}, e_{\mu}^{0}=\delta_{\mu}^{0}, e_{\mu}^{i}=a \delta_{\mu}^{i},
$$

where $a\left(x^{0}\right)$ is the scale factor, $x^{0}=c t$ and $\delta_{\mu}^{i}=\delta_{i \mu}$.

The affine connection

$$
\Gamma_{\mu \beta}^{\sigma}=\frac{1}{2} g^{\sigma \rho}\left(\partial_{\mu} g_{\beta \rho}+\partial_{\beta} g_{\rho \mu}-\partial_{\rho} g_{\mu \beta}\right)
$$

for this space time are given as (in the unit $c=\hbar=1$ )

$$
\Gamma_{0 \mu}^{0}=0, \quad \Gamma_{i j}^{0}=a \dot{a} \delta_{i j}, \quad \Gamma_{0 j}^{i}=\Gamma_{j 0}^{i}=\frac{\dot{a}}{a} \delta_{i j}, \quad \Gamma_{j l}^{i}=0 .
$$

Consequently, we can find

and also

$$
\sigma^{a} e_{a}^{\mu} \Gamma_{\mu}=\frac{3 \dot{a}}{2 a}
$$

$$
\sigma^{0} e_{0}^{\mu}-\sum_{k=1,2,3} \sigma^{k} e_{k}^{\mu}=\frac{3 \dot{a}}{2 a}
$$

Thus we have from (10)and (13), the Weyl equations as

$$
\sigma^{a} e_{a}^{\mu} \partial_{\mu} \psi^{(i)}+\frac{3 \dot{a}}{2 a} \psi^{(i)}=0
$$

and

$$
\left(\sigma^{0} e_{0}^{\mu}-\sum_{k=1,2,3} \sigma^{k} e_{k}^{\mu}\right) \partial_{\mu} \psi^{(i)}+\frac{3 \dot{a}}{2 a} \psi^{(i)}=0
$$

For $\psi^{(i)}$ dependent only on time we have the same Weyl equation for $\psi^{(i)}$ from (24)and (24 a)

$$
\sigma^{0} \partial_{0} \psi^{i}+\frac{3 \dot{a}}{2 a} \psi^{(i)}=0
$$

This equation can be identified with the equation (21) in which we take

$$
q\left(x^{0}\right)=\left[a\left(x^{0}\right)\right]^{-\frac{3}{2}}
$$

that is, ( in unit $c=\hbar=1$ )

$$
\frac{q^{\prime}\left(x^{0}\right)}{\left.q^{(} x^{0}\right)}=-\frac{3}{2} \frac{\dot{a}}{a}
$$

Therefore, the emerged Riemannian space time manifold due to the apparition of Weyl spinors which depend only on time and having symplectic property in the abstract spinor space must be the FRW space time (the cosmological space time). It is to be noted that this FRW spacetime is equivalent to the metric tensor

$$
g_{\mu \nu}=g\left(x^{0}\right) \eta_{\mu \nu}
$$

In fact, we can achieve this by a pure time transformation

$$
T=\int \frac{d t}{a(t)} \text { with } a(t) \equiv \sqrt{g(c T)}=\sqrt{g\left(x^{0}\right)}
$$

where $T$ is the cosmological time. Thus, the FRW space time (with flat space), the cosmological background space time of the universe,emerged.In this way, the gravity emerged spontaneously with Q M which is manifested in the quantum Weyl fields. 


\section{METRIC STRUCTURE: FINSLER SPACE}

We now present here an alternative construction of space time manifold with the metric structure from the two independent time dependent two component spinors. This consideration is similar to that of [13] and others [15-16] but it is also different in some respects. The two independent spinors are given in (11), from which the four vectors in Minkowski space time can be constructed. These are

$$
\begin{aligned}
& a^{m}=\psi^{(1) \dagger} \sigma^{m} \psi^{(1)}=\left[q\left(x^{0}\right)\right]^{2} \sigma_{11}^{m} \\
& b^{m}=\psi^{(2) \dagger} \sigma^{m} \psi^{(2)}=\left[q\left(x^{0}\right)\right]^{2} \sigma_{22}^{m} \\
& c^{m}=\psi^{(1) \dagger} \sigma^{m} \psi^{(2)}=\left[q\left(x^{0}\right)\right]^{2} \sigma_{12}^{m} \\
& d^{m}=\psi^{(2) \dagger} \sigma^{m} \psi^{(1)}=\left[q\left(x^{0}\right)\right]^{2} \sigma_{21}^{m}
\end{aligned}
$$

where $\sigma^{m}(\mathrm{~m}=1,2,3)$ are Pauli Matrices and $\sigma^{0}=1$.

Now, we construct space time tetrad differently from [13] and others in the following way.

$$
\left(e^{m \nu}=e^{\nu m}\right)=\left(\begin{array}{c}
e^{0 m} \\
e^{1 m} \\
e^{2 m} \\
e^{3 m}
\end{array}\right)=\left(\begin{array}{c}
\frac{1}{2} q^{2}\left(\sigma_{11}^{m}+\sigma_{22}^{m}\right) \\
\frac{1}{2} q^{2}\left(\sigma_{12}^{m}+\sigma_{21}^{m}\right) \\
\frac{-i}{2} q^{2}\left(\sigma_{12}^{m}-\sigma_{21}^{m}\right) \\
\frac{1}{2} q^{2}\left(\sigma_{11}^{m}-\sigma_{22}^{m}\right)
\end{array}\right)=\left(\begin{array}{c}
q^{2} \eta^{0 m} \\
q^{2} \eta^{1 m} \\
q^{2} \eta^{2 m} \\
q^{2} \eta^{3 m}
\end{array}\right)
$$

where $\eta_{\mu \nu}$ is the Minkowski metric tensor with signature $(+,-,-,-)$.

Thus

$$
e^{m \nu}=e^{\nu m}=q^{2} \eta^{m \nu}=q^{2} \eta^{\nu m} \text { and } e_{m}^{\mu}=q^{2} \eta_{m}=q^{2} \delta_{\mu m}
$$

Here, we get $e_{m}^{\mu}$ from $e^{m \nu}$ by transforming the spinors according to (5). We define a tensor $G^{\mu \nu}$ by

$$
G^{\mu \nu}=e_{m}^{\mu} e^{m \nu}
$$

Therefore, we have

$$
G^{\mu \nu}=q^{4} \eta^{\mu \nu}
$$

Now setting

$$
G^{\mu \nu} \equiv G=g g g \equiv\left(g^{\rho \sigma}\right)\left(g^{\rho \sigma}\right)\left(g^{\rho \sigma}\right),
$$

we get

$$
(g g g)^{\mu \nu}=q^{4} \eta^{\mu \nu}
$$

A solution of this equation is given by

$$
g^{\mu \nu}=q^{\frac{4}{3}} \eta^{\mu \nu}, \text { consequently, } g_{\mu \nu}=q^{-\frac{4}{3}} \eta_{\mu \nu}
$$

which is taken as the metric tensor constructed from the two independent spinors. By using the relations (26) and (29) we get

$$
g_{\mu \nu}=g\left(x^{0}\right) \eta_{\mu \nu}
$$

This metric tensor gives rise to the same geometric structure (given in (28)) of the manifold that emerged by the appearance of the Weyl spinors having the sympletic structure in the abstract spinor 
space. That is, the spinors of the flat space can produce the curved spacetime which is the background FRW universe being equivalent to the geometric structure (38). It will be a noteworthy fact that we can also arrive at a Finsler spacetime structure of the manifold apart from the Riemannian structure as above. In fact, the Finsler space that we shall get has the above space time with metric tensor (38) as the associated Riemannian spacetime of it. The fundamental function of the Finsler space is defined here as

$$
F^{4}=G_{\mu \nu \rho \sigma}(x) \nu^{\mu} \nu^{\nu} \nu^{\rho} \nu^{\sigma}
$$

or,

$$
d s^{4}=G_{\mu \nu \rho \sigma}(x) d x^{\mu} d x^{\nu} d x^{\rho} d x^{\sigma}
$$

The Finsler space introduced here is, in fact, in accord with Riemann's original suggestion that the positive fourth root of a fourth order differential form might serve as a metric function (Riemann,1854). Here we take

$$
G_{\mu \nu \rho \sigma}=G_{\mu \nu} G_{\rho \sigma}=q^{-8} \eta_{\mu \nu} \eta_{\rho \sigma}
$$

by using (34). Consequently, we have from (40)

$$
d s^{4}=q^{-8} \eta_{\mu \nu} \eta_{\rho \sigma} d x^{\mu} d x^{\nu} d x^{\rho} d x^{\sigma}=\left(q^{-4} \eta_{\mu \nu} d x^{\mu} d x^{\nu}\right)^{2}
$$

Now there are two possibilities from this relation :

i.e., the tensor $G_{\mu \nu}$ is given by

$$
d s^{2}=q^{-4} \eta_{\mu \nu} d x^{\mu} d x^{\nu}
$$

$$
G_{\mu \nu}=q^{-4} \eta_{\mu \nu}
$$

This leads to the Riemannian metric tensor (38).

(ii) On the other hand, we have

$$
d s^{2}=\theta\left(d x^{2}\right) q^{-4} \eta_{\mu \nu} d x^{\mu} d x^{\nu}
$$

where

$$
d x^{2}=\eta_{\mu \nu} d x^{\mu} d x^{\nu}
$$

and

$$
\theta(z)=1, z \geq 0 ; \theta(z)=-1, z<0
$$

From (43), we get a tensor

$$
G_{\mu \nu}=\theta\left(d x^{2}\right) q^{-4} \eta_{\mu \nu}
$$

Writing as before, $\left(G_{\mu \nu}\right) \equiv G=g g g$ and noting that

$$
\theta\left(d x^{2}\right)^{3}=\theta\left(d x^{2}\right)=\theta\left(\nu^{2}\right)
$$

where

$$
\nu^{\mu} \equiv \frac{d x^{\mu}}{d s}
$$

we have

$$
g_{\mu \nu}=\theta\left(\nu^{2}\right) g\left(x^{0}\right) \eta_{\mu \nu}
$$

and consequently,

$$
F^{2}=g_{\mu \nu} \nu^{\mu} \nu^{\nu}=\theta\left(\nu^{2}\right) g\left(x^{0}\right) \eta_{\mu \nu} \nu^{\mu} \nu^{\nu}=g\left(x^{0}\right) \theta\left(\nu^{2}\right) \nu^{2} .
$$

This is a Finsler space which was considered by [17] ( see also [18] ) as the anisotropic spacetime of hadronic matter extension. Here,this spacetime has been constructed from the tetrad fields by considering their correspondence with the two-dimensional spiner space endowed with a symplectic structure. It is to be noted that the Riemannian space time with the metric tensor (38) is the associated Riemannian manifold of the Finsler space. This Riemannian spacetime structure is the emergent gravity and it is, in fact, the cosmological background spacetime of the universe, as shown earlier. Once the structureless spacetime manifold transformed spontaneously into Finslerian manifold (with 
an associated Riemannian manifold), the spinor fields must consequently satisfy a field equation in that space. This equation has been proposed by [17] for the four-dimensional bispinors. The equation was derived from a classical field equation by the quantization of the space and time at the Planckorder scale. This quantization of space and time is also a consequence of the assumption of the minimum length and time. Also, the minimum length and time follow from the generalized uncertainty principle. We now discuss this GUP in next section.

\section{GENERALIZED UNCERTAINTY OPRINCIPLE:}

Modifications of the Heisenberg uncertainty principle near the planck scale are the GUP which are motivated by string theory, black hole physics,doubly special relativity and other theories of quantum gravity.Physical implications such as discreteness of space time have been considered by several authors [see [19] , [20] and references there in]. Here we examine the implication of GUP as proposed by [21] because in there the origin of the additional term making the modification has been discussed, and thus this can be regarded as independent of any model.They proposed the GUP as

$$
\delta x=\delta x_{H}+\delta x_{G}
$$

where

$$
\delta x_{H} \geq \frac{\hbar}{2 \delta p}
$$

and

$$
\delta x_{G} \geq \frac{2 L_{p}^{2} \delta p}{\hbar}
$$

where $\delta p$ is the momentum uncertainty of the electron and $L_{p}$ is the planck length. $\delta x_{H}$ is the Heisenberg uncertainty of position of electron and $\delta x_{G}$ is the additional term to the uncertainty of the position. Derivation of this additional term $\delta x_{G}$ has been made by them in four heuristic ways, two of which are based on Newtonian theory of gravitation where as the other two are on consideration of general relativity theory.

In fact, the position uncertainty of electron $\Delta x_{G}$ was shown to arise out from the gravitation interaction between photon as quanta and electron.It is to be noted that the electron momentum uncertainty $\Delta p$ must be of the order of interacting photon momentum $p=\frac{E}{c}=\frac{\hbar}{\lambda}$ where $E$ is the energy of photon and $\lambda$ is the photon wave length. Thus

$$
\delta p \simeq p=\frac{\hbar}{\lambda}
$$

Consequently, we have from (52),

$$
\delta x_{H} \geq \frac{\lambda}{2}
$$

This was Heisenberg's contention that one can not obtain better precision in the position of electron than $\lambda$ when measured with an electromagnetic wave of that wave length. That is the shorter wave length being responsible of good resolution cab make the position measurement of electron more precise. On the other hand, we have from (53) and (54),

$$
\delta x_{G} \geq \frac{L_{p}^{2}}{\lambda}
$$

Thus we see that with the shorter wave length $\lambda$ which corresponds to the higher energy and momentum of photon, the gravitational photon field interacting with electron makes its position less precise. Physically, such situation one should expect and can think that the additional uncertainty term $\Delta x_{G}$ is indeed, due to gravity and consequently related to the curved space time structure. 
Gao (2010), in a reverse application of GUP (51) of Adler and Santiago, has argued the energy and momentum of a particle can change the geometric structure of space time. There it is shown that the energy $E \simeq p c$ in a region with size $L$ can change the proper size of the region because of the positional uncertainty $\Delta x_{G}$ as in (53). The change is by an amount,

$$
\delta L \simeq \frac{L_{p} T_{p} E}{\hbar}
$$

where $T_{p}$ is the planck time. This implies that a flat space time transformed to the curved one by the energy momentum in the region.

Consequence of the GUP (51) is that the position uncertainty $\Delta x$ has a minimum

$$
\delta x_{\min } \simeq 2 L_{p}
$$

Also the similar uncertainty principle (for time uncertainty)

$$
\delta t \geq \frac{\hbar}{2 \Delta E}+\frac{2 T_{p}^{2} \Delta E}{\hbar}
$$

leads to a minimum time uncertainty,

$$
\delta t_{\text {min }} \simeq 2 T_{p}
$$

The implication of the minimum size of space time is that the interval shorter than planck scale is physically meaningless, that is the measurements can not be possible in interval shorter than this scale. In other words space time is quantized at the planck scale level [20].

Now in the present case, the structureless space time manifold is transformed spontaneously into a curved one, the cosmological background space time of the universe. Thus gravity emerged and due to GUP the space time became quantized with the minimum planck order length and time. On the contrary, the minimum length and time or quantized space time which is the consequence of QM can give rise to gravity due to emergent curved spacetime manifold. So it can be thought as a phenomenon of emergence of both QM and gravity. Here we see that it is due to the appearance of Weyl spiners dependent only on time in the flat spacetime manifold, and having a sympletic structure in the abstract spiner space.

\section{FIELD EQUATION AND MASSIVE PARTICLES:}

As in [17] the field equation for the two-dimensional 'bispinor' in the Finsler space can be derived for the massless case.(as also for massive bispinor fields). This equation for the bispinor $\Psi \equiv\left(\Psi_{\alpha \beta}\right)$ is (in the natural unit $\hbar=c=1)$

$$
i \sigma_{\alpha \beta^{\prime}}^{\mu}(x) \partial_{\mu} \Psi_{\beta^{\prime} \beta}(x, \nu) \mp i \sigma_{\beta \beta^{\prime}}^{\mu}(x) \gamma_{h \mu}^{l}(x, \nu) \nu^{h} \partial_{l}^{\prime} \Psi_{\alpha \beta^{\prime}}(x, \nu)=0,
$$

where $\gamma_{h \mu}^{l}(x, \nu)$ are Christoffel symbol of second kind ,and

$$
\partial_{\mu} \equiv \frac{\partial}{\partial x^{\mu}}, \quad \partial_{l}^{\prime} \equiv \frac{\partial}{\partial \nu^{l}} .
$$

Pauli matrices $\sigma^{i}(x)(\mathrm{i}=1,2,3)$ and $\sigma^{0}(x)$ are related to the flat space Pauli matrices $\sigma^{i}$ and $\sigma^{0}=1$ by the vierbein for the associated Riemannian manifold of the present Finsler space. The above equation (61) can be written as

$$
i \sigma_{\alpha \beta^{\prime}}^{\mu}(x)\left\{\partial_{\mu} \Psi_{\beta^{\prime} \beta} \mp \sigma_{\beta^{\prime} \nu}^{\mu^{-1}}(x) \sigma_{\beta \delta}^{\mu}(x) \gamma_{h \mu}^{l}(x, \nu) \nu^{h} \partial_{l}^{\prime} \Psi_{\nu \delta}\right\}=0,
$$


or,

$$
i \sigma_{\alpha \beta^{\prime}}^{\mu}(x) \partial_{\mu} \Psi_{\beta^{\prime} \beta}(x, \nu) \mp A_{\mu \beta^{\prime} \beta \nu \delta}^{l}(x, \nu) \partial_{l}^{\prime} \Psi_{\nu \delta}(x, \nu)=0,
$$

where

$$
A_{\mu \beta^{\prime} \beta \nu \delta}^{l}(x, \nu)=\sigma_{\beta^{\prime} \nu}^{\mu^{-1}}(x) \sigma_{\beta \delta}^{\mu}(x) \gamma_{h \mu}^{l}(x, \nu) \nu^{h} .
$$

That is, the equation can be written in compact form as

$$
i \sigma^{\mu} \nabla_{\mu} \Psi(x, \nu)=0,
$$

where

$$
\nabla_{\mu}=\partial_{\mu} \mp A_{\mu}^{l}(x, \nu) \partial_{l}^{\prime},
$$

is the generalized derivative. Now, for the Finsler space (50), $g_{\mu \nu}$ as in (49) is not Finslerian metric. On the other hand, the Finsler metric is given by

$$
\widehat{g}_{i j}=\frac{\partial^{2} F^{2}}{2 \partial \nu^{i} \partial \nu^{j}}
$$

The Christoffel symbols $\gamma_{h \nu}^{l}(x, \nu)$ are derived from the metric tensor $\widehat{g}_{i j}$ but it can be proved that the relation

$$
\gamma_{j k}^{i} \nu^{j} \nu^{k}=\widehat{\gamma}_{j k}^{i} \nu^{j} \nu^{k}
$$

holds good for the present Finsler space, where the Christoffel symbols $\widehat{\gamma}_{j k}^{i}$ are derived from $g_{i j}$. Because of this relation, we can use $\gamma_{j k}^{i}$ in the above field equation in stead of $\gamma_{j k}^{i}$. We can obtain $\gamma_{h \mu}^{l}$ as

$$
\widehat{\gamma}_{h \mu}^{l}=\frac{1}{2}\left[\delta_{h}^{l} \frac{\partial \ln g\left(x^{0}\right)}{\partial x^{\mu}}+\delta_{\mu}^{l} \frac{\partial \ln g\left(x^{0}\right)}{\partial x^{h}}-\eta^{l \beta} \eta_{h \mu} \frac{\partial \ln g\left(x^{0}\right)}{\partial x^{\beta}}\right] .
$$

The equation (63) retain its form with the flat space Pauli matrices $\sigma^{i}(\mathrm{i}=1,2,3)$ and $\sigma^{0}$ because of the vierbein for the spacetime (28), and therefore, using (68) we have

$$
\begin{gathered}
i \sigma^{\mu} A_{\mu}^{l}(x, \nu) \partial_{l}^{\prime} \Psi \equiv i \sigma_{\alpha \beta^{\prime}}^{\mu} A_{\mu \beta^{\prime} \beta \nu \delta}^{l} \partial_{l}^{\prime} \Psi_{\nu \delta}=\frac{i}{2} \sigma_{\alpha \beta^{\prime}}^{\mu} \sigma_{\beta^{\prime} \nu}^{\mu} \sigma_{\beta \delta}^{\mu T}\left[\nu^{l} \delta_{\mu}^{0}+\delta_{\mu}^{l} \nu^{0}-\eta^{l_{0}} \eta_{h \mu} \nu^{h}\right] \frac{d l_{n} g\left(x^{0}\right)}{d x^{0}} \sigma_{l}^{\prime} \Psi_{\nu \delta} \\
=\frac{i}{2} \delta_{\alpha \nu} \sigma_{\beta \delta}^{\mu T} \frac{g^{\prime}\left(x^{0}\right)}{g\left(x^{0}\right)}\left[\delta_{\mu}^{l} \nu^{0}-\eta^{l 0} \eta_{h \mu} \nu^{h}\right] \partial_{l}^{\prime} \Phi_{\delta} \Psi_{\nu}
\end{gathered}
$$

where the bispiner $\Psi_{\nu \delta}$ has been decomposed as

$$
\Psi_{\nu \delta}(x, \nu)=\Phi_{\delta}(\nu) \Psi_{\nu}(x)
$$

$\Phi_{\delta}(\nu)$ being a two dimensional spinor which is supposed to be a homogeneous function of $\nu=\left(\nu^{0}, \nu^{1}, \nu^{2}, \nu^{3}\right)$ of degree zero, i.e.,

$$
\Sigma_{l=1}^{3} \nu^{l} \partial_{l}^{\prime} \Phi_{\delta}(\nu)=0 .
$$

Then we have

$$
\mp i \sigma_{\alpha \beta^{\prime}}^{\mu} A_{\mu \beta^{\prime} \beta \nu \delta}^{l} \partial_{l}^{\prime} \Psi_{\nu \delta}=\mp \frac{i}{2} \Psi_{\alpha} \frac{d l_{n} g\left(x^{0}\right)}{d x^{0}} \Sigma_{l=1}^{3} \sigma_{\beta \delta^{i T}}\left(\nu^{0} \partial_{i}+\nu^{i} \partial_{0}\right) \phi_{\delta}=\frac{3 i g^{\prime}\left(x^{0}\right)}{4 g\left(x^{0}\right)} \Psi_{\alpha} \Phi_{\beta},
$$

if

$$
\Sigma_{l=1}^{3} \sigma_{\beta \delta}^{i T}\left(\nu^{0} \partial_{i}+\nu^{i} \partial_{0}\right) \Phi_{\delta}(\nu)=\mp \frac{3}{2} \phi_{\beta}(\nu) .
$$

Therefore, from (65), (66) and (72) we have

$$
\sigma_{\alpha \beta^{\prime}}^{\mu} \partial_{\mu} \Psi_{\beta^{\prime}}(x) \Phi_{\beta}(\nu)+\frac{3 g^{\prime}\left(x^{0}\right)}{4 g\left(x^{0}\right)} \Psi_{\alpha}(x) \Phi_{\beta}(\nu)=0,
$$

or,

$$
\sigma_{\alpha \beta^{\prime}}^{\mu} \partial_{\mu} \Psi_{\beta^{\prime}}(x)+\frac{3 g^{\prime}\left(x^{0}\right)}{g\left(x^{0}\right)} \Psi_{\alpha}(x)=0
$$


That is,

$$
\sigma^{\mu} \partial_{\mu} \Psi(x)-\frac{3 g^{\prime}\left(x^{0}\right)}{4 g\left(x^{0}\right)} \Psi(x)=0 .
$$

This equation is the same as (21) for the time dependent spinors. Also, we can have Dirac equation for massive spiner field in the associated Riemannian spacetime manifold of the Finsler space as in De (2003). The field equation for the bispiner $\Psi(x, \nu)=\Psi_{\alpha \beta}(x, \nu)$ in the Finsler space is

$$
i \hbar \gamma_{\alpha \beta^{\prime}}^{\mu}(x) \partial_{\mu} \Psi_{\beta^{\prime} \beta}(x, \nu)-\gamma_{\beta \beta^{\prime}}^{\mu}(x) \gamma_{h \mu}^{l}(x, \nu) \nu^{h} \partial_{l}^{\prime} \Psi_{\alpha \beta^{\prime}}(x, \nu)=m c \Psi_{\alpha \beta}(x, \nu),
$$

where $\gamma_{\alpha \beta^{\prime}}^{\mu}(x)$ are related to the flat space Dirac matrices $\gamma^{\mu}$ by the vierbein for the spacetime metric tensor $(28)$.

With the decomposition

$$
\Psi(x, \nu)=\Psi_{1}(x) \Phi^{T}(\nu)+\Psi_{2}(x) \Phi^{c T}(\nu)
$$

where $\Psi_{1}$ and $\Psi_{2}$ are eigenstates of $\nu^{0}$ with eigenvalues +1 and -1 respectively, we have the following Dirac equation for $\Psi(x, \nu)$ :

$$
i \hbar\left(\gamma^{\mu} \partial_{\mu}-\frac{3 b_{0}}{2} \xi(t) \nu^{0}\right) \Psi(x, \nu)=\frac{c}{e(t)}(m+M \xi(t) e(t)) \Psi(x, \nu),
$$

if $\Phi(\nu)$ and $\Phi^{c}(\nu)$ satisfy the following equations

$$
\begin{array}{r}
i \hbar \gamma^{\mu} \gamma_{h \mu}^{l} \gamma^{h} \partial_{l}^{\prime} \Phi(\nu)=\left(M c-\frac{3 i \hbar b_{0}}{2}\right) \Phi(\nu), \\
i \hbar \gamma^{\mu} \gamma_{h \mu}^{l} \nu^{h} \partial_{l}^{\prime} \Phi^{c}(\nu)=\left(M c+\frac{3 i \hbar b_{0}}{2}\right) \Phi^{c}(\nu) .
\end{array}
$$

If $\Phi(\nu)$ and $\Phi^{c}(\nu)$ are homogeneous function of $\nu=\left(\nu^{0}, \nu^{1}, \nu^{2}, \nu^{3}\right)$ of degree zero , then the equations (78) and (78a) become

$$
\begin{gathered}
i \hbar b_{0} \Sigma_{l=1}^{3} \nu^{l}\left(\nu^{l} \frac{\partial}{\partial \nu^{0}}+\nu^{0} \frac{\partial}{\partial \nu^{l}}\right) \Phi(\nu)=-\left(M c-\frac{3 i \hbar b_{0}}{2}\right) \Phi(\nu), \\
i \hbar b_{0} \Sigma_{l=1}^{3} \nu^{l}\left(\nu^{l} \frac{\partial}{\partial \nu^{0}}+\nu^{0} \frac{\partial}{\partial \nu^{l}}\right) \Phi^{c}(\nu)=-\left(M c+\frac{3 i \hbar b_{0}}{2}\right) \Phi^{c}(\nu) .
\end{gathered}
$$

Here,

$$
e(t)=\frac{1}{\sqrt{g\left(x^{0}\right)}}, \quad 2 b_{0} \xi(t)=\frac{g^{\prime}\left(x^{0}\right)}{g\left(x^{0}\right)},
$$

and $\mathrm{m}$ is the inherent mass of the spinor. Also, here $\mathrm{M}$ appears as a constant in the process of separation of the field equation (75). $\Psi(x, \nu)$ satisfies the Dirac equation $(77)$ in the associated Riemannian space which is, in fact, a space time conformal to Minkowski flat space time.The Dirac equation for FRW spacetime can be obtained by the pure time transformation (29). If $M \neq 0$, the additional mass term is time dependent. The mass of the spinor is given by

$$
m+M \xi(t) e(t)=m+\frac{M}{b_{0} c} H(T)
$$

where

$$
H(T)=\frac{R^{\prime}(T)}{R(T)},
$$

is the Hubble's function.For the case $\mathrm{M}=0$, it can be seen that the $\nu$ dependent spiners $\Phi(\nu)$ and $\Phi^{c}(\nu)$ in the decomposition of $\Psi(x, \nu)$ can give rise to an additional quantum number if it is the field of the constituent-particle in the hadron configuration. This quantum number can generate the 
interval symmetry of hadrons $[18,22]$. The parameter $b_{0}$ of the spacetime in the epoch dependent mass term in (81) is found to be connected with $\mathrm{M}$. This relation has been obtained in the derivation of solutions for $\phi(\nu)$ and $\phi^{c}(\nu)$ of the equations of 79 and 79(a) [17]. It is shown there that $M$ must be proportional to $b_{0}$ as follows:

$$
(0.832) \hbar b_{0}=M c
$$

Then the mass term in (81) becomes

$$
m+(0.832) \frac{\hbar}{c^{2}} H(T)
$$

Then the parameter $b_{0}$ is in fact connected to the expansion of the universe.

For example $g\left(x^{0}\right)=\left(b_{0} x_{0}\right)^{4}$. The scale factor and Hubble's function respectively found to be

and

$$
R(T)=\left(3 b_{0} c T\right)^{\frac{2}{3}}
$$

$$
H(T)=\frac{2}{3 T}
$$

Thus we have an epoch dependent mass term apart from the inherent mass of the spinor even for massless particles like neutrinos. This epoch dependent mass which is of the order of planck mass at the Planck order time (the minimum time as obtained from GUP) becomes negligible at the present epoch of the universe. These highly massive particles give rise to the gravitation by fulfilling the Einstein equivalence with their mass distribution in the emerged curved space time. The inherent masses of the particles might be achievable from self intersection phenomenon or otherwise [23]. Actually these highly massive particles can deliver necessary mass density in the very early universe [24].

\section{THE SCENARIO AND CONCLUDING REMARKS}

We have seen that due to the emerged gravity the Heisenberg uncertainty principle is to be modified into GUP, which in turn lead to the discreteness of space time with the minimum time and length uncertainty, and vice versa. As an implication of the discreteness of the space time or consequent GUP, Gao [25] also has shown that flat space becomes curved by the energy momentum contained in it. This provides a deeper meaning for the Einstein Equivalence principle in G R. On the basis of this principle it is there pointed out that Einstein field equation can be derived by imposing some reasonable conditions on the Riemannian symmetric second rank tensor constructed from the metric of the space time. and its derivative and on energy momentum (i.e, the conservation of energy and momentum) i.e., the emerged curved manifold together with the highly massive particles there in all emerged instantaneously and simultaneously with the Quantum Weyl particles dependent only on time and with the symplectic conditions satisfied by them in the abstract spinor space can be regarded as the origin of the expanding universe which is in fact, the associated Riemannian manifold of the emerged Finsler space. In a sense, both Q M and gravity as derived by G R are fundamental as well as emergent. The phenomenon of emergence rather, the unified emergence occurs instantaneously. The instantaneousness is in fact, the minimum time (as obtained from GUP) which is of the order of Planck time and the fact is that it is indivisible. Thus the minimum time is only conceivable as zero time or instantaneous. After that Planck order time, the time may be regarded as continuous because we need not have to consider any phenomenon in later time in the minimum inconceivable and indivisible discrete time.

In the scenario described above we see a parameter $b_{0}$ which is related with the space time structure. This parameter specifies the time dependence of Weyl spinors as well as consequent structure of the emergent space time manifold. Obviously it appears as the scale factor of the expanding universe. Therefore it determines the age of the universe from the scale factor of FRW space time. Again we see that $b_{0}$ is linearly related to the mass term parameter $M$. Therefore $M$ is not a new parameter. On the other hand, if $M$ is taken as to be Planck order mass, then $b_{0}$ is fixed by it, and the age of the universe is specified.Thus $b_{0}$ and $M$ are no new parameters, they are derivable from the fundamental 
constants $\hbar, c$ and the gravitational constant $G$.

Finally we like to say that the gravity may be quantized in the present framework because the metric structure of the space time is here constructed from the spinors which can be subjected to quantization as in Q M.

\section{Acknowledgments}

FR is thankful to the Inter-University Centre for Astronomy and Astrophysics (IUCAA), India for providing Visiting Associateship. We are thankful to the referee for his comments. FR is also grateful to the DST, Govt. of India, for financial support under PURSE programme.

\section{References}

[1] L. Smolin , Three roads to quantum gravity, Oxford University Press (2000).

[2] C. Rovelli , Quantum Gravity, Cambridge (2004).

[3] C. Kiefer, Quantum gravity, Oxford University Press (2004).

[4] L.J. Garay, Int.J.Mod.Phys. A10, 145 (1995).

[5] M . Gasperini and G. Veneziano, Phys. Rept., 373, 1(2003).

[6] A. Ashtekar, M. Bojowald and J. Lewandowski , Adv.Theor.Math.Phys. 7, 233 (2003).

[7] A. Ashtekar, Class.Quan.Grav. , 21 , R53 (2004).

[8] A. Ashtekar, AIP Conf.Proc. 1241 , 109 (2010)

[9] M. Bojowald, J. Phys.: Conf. Ser. 405 , 012001 (2012).

[10] N. Birrel and P. Davies, Quantum Fieldsa in Curved Space, Cambridge University Press (1982).

[11] Yi-Fu. Cai, M. ZLi and X. Zhang, Physics Letters B 718 , 248 (2012).

[12] A.O. Barut and L.P. Singh, Int.J.Mod.Phys. D 4, 479 (1995).

[13] M. Kober, Phys.Rev.D79, 084009 (2009).

[14] E. Cartan, The theory of spinors, Dover publication (1981).

[15] R. Penrose and W. Rindler, Spinors and Spacetime (Vol-I), Cambridge University Press, U.K (1984).

[16] R. Penrose and W. Rindler, Spinors and Spacetime (Vol-II), Cambridge University Press, U.K (1986).

[17] S.S. De, arxiv:hep-th/0308180 (2003).

[18] S.S. De and F. Rahaman, Finsler Geometry of Hadrons and Lyra Geometry : Cosmological Aspects, Lambert Academic Publishing, Germany (2012).

[19] A.F. Ali, S. Das and E.C. Vagenas, Phys.Lett.B 678, 497 (2009).

[20] A.A. Abutaleb, Adv.High Energy Phys. 2013 , 124543 (2013).

[21] R.J. Alder, and D.I. Santiogo, Mod.Phys.Lett.A 14, 1371 (1999). 
[22] S.S. De, Int.Jour.Theor.Phys.(36), 89 (1997).

[23] J. Hansson, Prog.Phys. 10 , 71 (2014).

[24] S.S. De, Int.Jour.Theor.Phys.(40)2067 (2001).

[25] S. Gao, Entropy 13, 936 (2011) . 